\title{
Factores de excelencia y prácticas escolares desde la perspectiva de los discentes
}

Factors of Excellence and School Practices from the Perspective of Students

Facteurs d'excellence et pratiques scolaires depuis la perspective des apprenants

Fatores de excelência e práticas escolares desde a perspectiva dos discentes

Fecha de recepción: 27 DE JULIO DE 2016/Fecha de aceptación: 25 DE JULIO DE 2017/Fecha de disponibilidad en línea: 1 DE DICIEMBRE DE 2017

Encuentre este artículo en http://magisinvestigacioneducacion.javeriana.edu.co/

\section{Resumen}

Este artículo de investigación analiza las percepciones sobre los factores de excelencia que son compartidas por estudiantes de seis liceos públicos de alto rendimiento de Santiago de Chile y reflexiona también acerca de las configuraciones de sus prácticas de estudio para lograr el éxito. Los análisis estadísticos descriptivos y factoriales de las encuestas y los análisis de contenido de las entrevistas grupales permitieron concluir que los alumnos asocian los factores del éxito con dimensiones expresivas, como el bienestar en el colegio, y presentan una fuerte adhesión al proyecto escolar, al gestionar de forma eficaz los tiempos de estudio y de ocio.

\section{Palabras clave}

Educación formal; calidad de la educación;

enseñanza pública; aprendizaje activo

Para citar este artículo / To cite this article / Pour citer cet article / Para citar este artigo

Quaresma, Maria Luísa (2017). Factores de excelencia y prácticas escolares desde la perspectiva de los discentes. magis, Revista Internacional de Investigación en Educación, 10 (20), 183-200. https://doi.org/10.11144/Javeriana.m10-20.fepe 


\section{Keywords}

Formal education; quality of education; public education; active learning

\section{Mots clés}

Éducation formelle; qualité de l'éducation; enseignement publique; apprentissage actif

\section{Abstract}

This article analyzes the perceptions about the factors of excellence that are shared by students from six public schools of Santiago de Chile, also reflecting on the configurations of their study practices to achieve success. The descriptive and factorial statistical analyzes of the surveys and the content analyzes of the group interviews allowed us to conclude that students associate success factors with expressive dimensions, such as well-being in the school, and they have a strong adherence to the school project, managing effectively their study and leisure time.

\section{Résumé}

Cet article de recherche analyse les perceptions par rapport aux facteurs d'excellence que sont partagées par les étudiants de six lycées publics de haut rendement de Santiago au Chili, on réfléchit aussi par rapport aux configurations de leurs pratiques d'étude pour réussir. Les analyses statistiques, descriptives et factorielles des enquêtes et les analyses de contenu des entretiens de groups nous ont permis conclure que les élèves font un lien entre les facteurs de succès et les dimensions expressives, tels que le bien-être au lycée, et présentent une forte adhésion au projet scolaire, en gérant de façon efficace les temps d'étude et les loisirs.

\section{Palavras-chave}

Educação formal; qualidade da educação; ensino público; aprendizagem ativa

\section{Resumo}

Este artigo de pesquisa analisa as percepções sobre os fatores de excelência que são compartilhadas por estudantes de seis liceus públicos de alto rendimento de Santiago do Chile, refletindo também a respeito das configurações de suas práticas de estudo para conseguir o sucesso. As análises estatísticas descritivas e fatoriais dos questionários e as análises de conteúdo das entrevistas grupais permitiram concluir que os alunos associam os fatores do sucesso com dimensões expressivas, como é o bem-estar no colégio, e apresentam uma forte adesão ao projeto escoar, gerenciando de forma eficaz os tempos de estudo e de lazer. 


\section{Introducción}

La promoción de la excelencia, actualmente indisociable de la promoción de la equidad (Duru-Bellat \& Mingat, 2011), ha ganado importancia como consecuencia de la permeabilidad del campo educativo a las lógicas de accountability (rendición de cuentas), en un escenario de crisis de confianza en la calidad de los sistemas públicos de educación (Maroy, 2012). Sin embargo, la calidad de este sector —así como la del sector privado- no es homogénea (Van Zanten, 2009): hay escuelas públicas que han sido reductos de excelencia académica, como un conjunto de liceos públicos de alto rendimiento en Santiago de Chile, comúnmente conocidos como Liceos Emblemáticos. En un sistema basado en la competencia y organizado en torno a la idea de mercado educativo, como el chileno, el crecimiento del sector privado y los procesos de segmentación socioeconómica de los establecimientos favorecen una desigual distribución de los estudiantes: las escuelas públicas acogen a estudiantes vulnerables, las particulares subvencionadas a los estudiantes de nivel medio bajo, medio y medio alto y los particulares pagados a los estudiantes de la élite. Lo anterior constituye un rasgo que transforma a Chile en un caso particular en el concierto internacional (Quaresma, 2015). En este contexto, los liceos públicos de alto rendimiento constituyen la excepción de calidad en el marco de la educación pública, aunque lograda, según autores como Juan Pablo Valenzuela y Claudio Allende-González (2012), por medio de procesos de "descreme" de los mejores alumnos. Además de los procesos de selección realizados en el momento de ingreso al colegio, estas escuelas se diferencian de los demás establecimientos públicos por un conjunto de rasgos que les confieren un estatuto de distinción: la tradición y el prestigio, la defensa del ideal republicano de instruir y preparar ciudadanos para glorificar a la patria, los altos niveles de rendimiento académico en las pruebas de selección universitaria, el reconocimiento social como escuelas de calidad y la casi inexistencia de coeducación de género.

Este artículo pretende reflexionar sobre estos colegios públicos de excelencia y poner el foco en sus alumnos: en sus percepciones sobre los factores que contribuyen para la construcción del éxito escolar y en sus prácticas individuales de estudio. El documento está organizado en cuatro acápites además de este. En el primero se presentan los principales hallazgos teóricos sobre los factores del éxito y, en el segundo, se dan a conocer las técnicas y procedimientos metodológicos. En el ámbito de la discusión de resultados se identifican los orígenes sociales de los alumnos y se reflexiona sobre las dinámicas de reproducción/movilidad social, se analizan las representaciones sociales de los estudiantes sobre los factores de excelencia y se presentan las prácticas diarias de estudio de estos alumnos, para discutir la importancia asumida por la familia y escuela en las tareas académicas de los jóvenes. En el último acápite se debaten las principales conclusiones.

\section{Referentes conceptuales}

Uno de los temas más relevantes en el estudio del éxito escolar, al cual la literatura ha dado especial relevancia, se relaciona con los factores que lo determinan o influyen. La familia está entre los factores que reúnen más consenso (Duru-Bellat, 2010; Schneider, Keesler \& Morlock, 2010). Una de las variables familiares más señaladas desde los estudios de Pierre Bourdieu y Jean-Claude Passeron (1964) ha sido la pertenencia social, generadora de desigualdades sociales ante la escuela (Duru-Bellat, 2010)
Descripción del artículo | Article description | Description de l'article | Artigo descrição

En este artículo de investigación, la autora reflexiona, por un lado, sobre las percepciones de los estudiantes de seis liceos públicos de alto rendimiento de Santiago de Chile sobre los factores de excelencia y, por otro lado, sobre la adhesión de estos estudiantes al proyecto escolar, y discute las configuraciones de sus prácticas de estudio y el rol de los agentes socializadores en el acompañamiento de sus actividades escolares. En este artículo se presentan los resultados del proyecto de La excelencia académica en escuelas públicas de prestigio: percepciones, vivencias y prácticas de los actores escolares, una investigación más amplia, financiada por la Comisión Nacional de Investigación Científica y Tecnológica, CONICYT (CONICYT, Fondo Nacional de Desarrollo Científico y Tecnológico, FONDECYT Iniciación número 11140241). Maria Luísa Quaresma, investigadora de la Universidad Autónoma de Chile, ejerce la función de investigadora responsable de este estudio. 
que tienen fuerte expresión en Chile, uno de los países con participación en las pruebas del Programme for International Student Assessment (PISA) "donde los factores socioeconómicos familiares tienen el mayor peso para explicar los logros educacionales de los alumnos" (Bellei, 2015, p. 182). De acuerdo con esta perspectiva, las clases económicas más privilegiadas se benefician de numerosas ventajas para que los hijos logren la excelencia escolar, como la posesión de capital cultural. Como ya fue documentado, hay una correlación positiva entre éxito escolar y escolaridad de los padres —en particular, de las madres_, a quienes compete asegurar el bienestar de los hijos y su desarrollo cognitivo-cultural (Gubbins-Foxley, 2014; Henri-Panabière, 2010). La capitalización cultural de las familias potencia no solamente la fluidez de la interacción con la escuela y con sus profesores (Lareau, 2011), sino también un acompañamiento en casa de las tareas escolares, que se reviste, además, de un carácter pedagógico (Lahire, 2008; Quaresma, 2013), y constituye una indiscutible ventaja escolar sobre el tipo de acompañamiento que las familias populares pueden dar a sus hijos. Estas, aunque no dimitan de la función de acompañar a sus hijos en las tareas escolares, no van más allá de la mera vigilancia sobre el cumplimiento de las tareas y/o sobre su presentación estética, lo que se explica, principalmente, por sus reducidas competencias académicas (Lareau, 2011).

Los recursos económicos familiares también son esenciales en la definición de trayectorias escolares exitosas, pues posibilitan el acceso a bienes y servicios escolarmente rentables: el capital cultural objetivado, sobre todo en forma de libros, material pedagógico extra y clases particulares de apoyo (Glasman, 2014; Gubbins-Foxley, 2014), y también las actividades de ocio cultivado (Lareau, 2011). No menos importante es la posibilidad económica de realizar elecciones residenciales que garanticen el acceso de los hijos a las mejores escuelas públicas (Van Zanten, 2015) o a los mejores y más selectivos colegios particulares que forman a las élites académicas y sociales (Gessaghi \& Méndez, 2015; Quaresma, 2014).

Los capitales informacionales y relacionales también confieren a las familias ventajas adicionales en el diseño de las trayectorias académicas de sus hijos. Las familias más informadas, debido a su manejo de información, pueden realizar una elección criteriosa del colegio que se ajuste a su proyecto formativo y al perfil de los niños (Gubbins-Foxley, 2014; Van Zanten, 2009). Las familias con mayores niveles de capital relacional tienen más facilidad en superar eventuales obstáculos de acceso a colegios con la calidad académica y social deseada (Van Zanten, 2009), lo cual alimenta las desigualdades sociales de acceso al éxito. Esto se puede observar en Santiago de Chile, ciudad que presenta un fuerte nivel de segregación socioeconómica y también escolar, siendo el más elevado entre los 57 países participantes en la prueba PISA 2006 (Valenzuela, Bellei \& De Los Ríos, 2010) y que fue alimentado por la lógica de la mercantilización de la educación (Bellei, 2015). En un contexto marcado por el aumento de los “(...) procesos de fragmentación y diferenciación social y académica entre los estudiantes y en la sociedad" (Villalobos \& Valenzuela, 2012, p. 165), más del $93 \%$ de los estudiantes chilenos del quintil más alto de ingreso asisten a escuelas particulares pagadas y más del $80 \%$ de los alumnos de los quintiles más bajos estudian en escuelas municipales (Assaél-Budnik, Cornejo-Chávez, González-López, Redondo-Rojo, Sánchez-Edmonson \& Sobarzo-Morales, 2011).

Sin embargo, estudios más recientes han relativizado el peso del determinismo social, que da cuenta de la existencia de trayectorias escolares "socialmente improbables", como el caso del fracaso escolar de los "herederos" (Henri-Panabière, 2010; Nogueira, 2004) y del éxito escolar de los 
jóvenes de las clases populares (Castets-Fontaine, 2015; Lahire, 2008), lo que ha develado la posibilidad de que la trayectoria escolar pueda asociarse a otras variables, relativamente autónomas con respecto a la clase social, como las características "personales" de los estudiantes, el colegio al que asisten, la formación científica y pedagógica de los docentes 0 , incluso, las dinámicas internas de las familias (Nogueira, 2004).

Se abren, así, nuevas pistas analíticas. Ellen S. Amatea, Sondra SmithAdcock y Elizabeth Villares (2006), por ejemplo, destacan el rol del estilo educativo de la familia, al cual atribuyen mayor peso que a la variable clase social. Annette Lareau (2011) también identifica estilos parentales más favorables al éxito escolar que se destacan por la organización de actividades de ocio, el desarrollo de la racionalización y negociación, el uso del lenguaje y la valoración de las opiniones y habilidades de los niños. El desarrollo de la autoconfianza, del espíritu crítico y de la capacidad de trabajo y organización en un clima de valoración, comprensión y diálogo también están asociados al éxito escolar (Kellerhals \& Montandon, 1991), junto con el incentivo al autocontrol y a la autonomía (Martín-Criado, Gómez-Bueno, Fernández-Palomares \& Rodríguez-Monge, 2000), la elevada exigencia y afecto, y la valoración de la igualdad de trato y de la responsabilidad (Gubbins-Foxley, 2014).

Otro elemento que también puede hacer la diferencia en la construcción del éxito escolar es la escuela, como plantea el Movimiento de las Escuelas Eficaces. Pero es el profesor quien tiene el papel principal en ese proceso (Darling-Hammond, 2000); su calidad se considera un factor determinante del aprendizaje (Bruns \& Luque, 2014), esencialmente en términos de su formación científica (Darling-Hammond, 2000). Esta dimensión todavía presenta falencias significativas en Chile, si tenemos en cuenta que menos del 3\% de los docentes ha obtenido la nota de "excelente" en las evaluaciones de conocimientos sobre las materias (Bruns \& Luque, 2014).

En cuanto a las prácticas pedagógicas, la literatura señala que el profesor eficaz es el que planifica adecuadamente las clases y mantiene un clima de tranquilidad y de respeto mutuo (Bellei, Valenzuela, Vanni \& Contreras, 2014), elige el método adaptado a contextos y objetivos, diversifica sus actividades, gestiona correctamente el tiempo de aprendizaje, diagnostica regularmente los progresos de los alumnos, e incentiva la cooperación con sus colegas (Bolívar, 2012), y además es capaz de mostrar flexibilidad y apertura a sus ideas, para estimular sus iniciativas (Bressoux, 2012) y establecer fácilmente comunicación con ellos (Brunner \& Elacqua, 2003). Las competencias comunicativas (Darling-Hammond, 2000) y también relacionales y emocionales (Barrère, 2002) facilitan la relación de cercanía y confianza favorable al éxito; entonces, es relevante que el profesor verbalice sus altas expectativas sobre el desempeño de sus alumnos, refuerce positivamente sus progresos, haga críticas constructivas y los motive constantemente (Bressoux, 2012).

En cuanto a las características personales del alumno consideradas fundamentales para la construcción del éxito escolar, investigaciones desarrolladas con estudiantes exitosos de la enseñanza media y superior (Álvarez-Rojo, García-Jiménez, Gil-Flores \& Romero-Rodríguez, 1999; Nogueira, 2004; Palhares \& Torres, 2011; Vieira, 2014) identifican jóvenes responsables, con fuerte autoestima, con una elevada motivación, persistentes, perfeccionistas y autocríticos. Ellos definen sus objetivos y metas y planifican, monitorean y evalúan sus actividades. Por otro lado, Shamus Rahman Khan (2011) habla de jóvenes con una ética de trabajo y de esfuerzo personal conforme a la narrativa de la meritocracia alimentada por la escuela y que encuentra fuerte adhesión en la sociedad chilena (Araujo \& Martuccelli, 2015). 
Adicionalmente, también se ha observado que la capacidad para memorizar, asimilar los contenidos, aclarar dudas o mantener un buen vínculo con sus profesores son características de los alumnos exitosos, quienes, además, atribuyen su éxito a su empeño diario en las clases, al estudio en casa y a la ayuda de profesores y de las nuevas tecnologías de información (Palhares \& Torres, 2011). Se destacan como importantes estrategias de estudio la elaboración de resúmenes y de esquemas, la consulta de manuales y la resolución de ejercicios, y también la adquisición de rutinas de trabajo. En cuanto a la dimensión temporal, la capacidad de hacer una gestión eficaz del tiempo, que es objeto de socialización en las escuelas de formación de las élites académicas (Darmon, 2015), es fundamental para lidiar con la "sobrecarga de trabajo" (p. 145) y las presiones para la urgencia que caracterizan la carrera de "alta competencia" (Vieira, 2014) en que se ha transformado la trayectoria hacia la excelencia. Ni el entorno familiar ni otro factor pueden predecir de forma aislada esta meta que será, pues, reflejo de la interacción de múltiples factores que operan en "círculo virtuoso" (Castets-Fontaine, 2011).

\section{Metodología}

Los datos presentados resultan de una investigación sobre la excelencia en escuelas públicas de alto rendimiento y prestigio. En este proyecto se aplicaron técnicas de recopilación de datos cuantitativos y cualitativos a los agentes de las comunidades educativas (directivos, profesores, alumnos y apoderados) de seis liceos públicos de alto rendimiento de la región metropolitana de Santiago de Chile. En el proceso de selección de los colegios se definieron algunos requisitos, como: (1) que fueran escuelas con mínimo de 50 años de existencia; (2) que fueran socialmente reconocidas por su prestigio y calidad; (3) que tengan proyectos educativos marcados por los ideales de orgullo de pertenencia al colegio y de logro académico; (4) que desde 2010 hayan formado parte del grupo de escuelas públicas con mejor rendimiento en la Prueba de Selección Universitaria (PSU); (5) que sus sedes estén ubicadas en tres comunas distintas social y culturalmente; y, (6) que sus asistentes sean de distintos públicos en términos de género (mixtos, masculinos y femeninos).

Tabla 1

Caracterización de los liceos emblemáticos

\begin{tabular}{l|l|l|l|l|l}
\hline Liceo & Fundación & Lema & $\begin{array}{l}\text { Matriculados } \\
\mathbf{( 2 0 1 6 )}\end{array}$ & Público & Comuna \\
\hline Liceo 1 & 1813 & Labor omnia vincit & 4.127 & Masculino & Santiago \\
\hline Liceo 2 & 1961 & $\begin{array}{l}\text { Con responsabilidad y tolerancia, } \\
\text { el Liceo (...) crea futuro }\end{array}$ & 1.751 & Femenino & Providencia \\
\hline Liceo 3 & 1942 & El estudio es la ruta mejor & 1.328 & Femenino & Providencia \\
\hline Liceo 4 & 1913 & $\begin{array}{l}\text { Ardiendo la llama del } \\
\text { conocimiento }\end{array}$ & 2.608 & Masculino & Providencia \\
\hline Liceo 5 & 1955 & $\begin{array}{l}\text { De compromiso protagónico } \\
\text { integral, APRENADEIM }\end{array}$ & 719 & Mixto & Nuñoa \\
\hline Liceo 6 & 1892 & Mens sana in corpore sano & 1.985 & Masculino & Santiago \\
\hline \hline
\end{tabular}

Fuente: elaboración propia 
En este artículo se analizarán solamente los datos relativos al alumnado. Estos fueron recopilados por medio de una encuesta y de entrevistas de grupo. La encuesta fue aplicada a una muestra de 1.828 estudiantes de tercer y cuarto medio (alumnos con edades comprendidas entre los 16 y 18 años), respetando la diversidad de electivos que tiene cada establecimiento. Los cursos fueron elegidos aleatoriamente y se encuestaron dos cursos por electivo, por nivel (seis cursos de tercero y seis de cuarto, por colegio). Este criterio no fue respetado en el liceo 5 (que solamente cuenta con un curso por nivel; por lo tanto, se encuestó a la totalidad de los estudiantes) y en el liceo 2, cuyos directivos no autorizaron la encuesta por cursos y exigieron la selección de una muestra aleatoria de estudiantes. Todos los estudiantes contestaron satisfactoriamente la encuesta, no se registró ningún caso de encuesta anulada. De los alumnos encuestados, fueron seleccionados 97 de manera aleatoria para participar en grupos focales. Los estudiantes fueron organizados en grupos de cuatro o cinco miembros y se realizaron cuatro grupos por colegio. Los alumnos que rechazaron la participación en los grupos focales fueron reemplazados por otros estudiantes, también seleccionados aleatoriamente. Los grupos focales tuvieron una duración de una hora, aproximadamente, y se discutieron temas como las percepciones de los estudiantes sobre la excelencia, los significados atribuidos a la meritocracia, sus vivencias y percepciones sobre el colegio - específicamente sobre las exigencias y expectativas depositadas en ellos por parte de directivos y profesores-, su vida extraescolar y gestión de los tiempos libres, sus motivaciones para la elección del colegio y, finalmente, sus percepciones sobre su pertenencia a la élite académica de Chile y sobre los rasgos que los diferencian relativamente los demás estudiantes, sean de colegios municipales o particulares. La discusión sobre estas temáticas permite reflexionar sobre las percepciones y experiencias de alumnos exitosos en un sistema educativo fuertemente jerarquizado y segregado, como el chileno, y que ha servido de ejemplo a países que se vienen abriendo a la mercantilización de la educación.

Los datos recopilados en la encuesta fueron tratados con el Statistical Package for Social Science (IBM SPSS, versión 20 en español) y los contenidos recogidos en las entrevistas fueron analizados mediante el software de análisis cualitativo NVivo.

En cuanto a los análisis estadísticos que dieron origen a los índices y clusters relativos a las percepciones sobre los factores de excelencia, cabe especificar los procedimientos seguidos. En primer lugar, se realizó una inspección de las variables con el alpha de Cronbach, un preprocesamiento de las variables que permite trabajar con un pool no demasiado grande in- dependiente de la dimensionalidad del índice final, y comprobar que las variables seleccionadas obtuvieran un Alpha mayor o igual a 0,7 y tuvieran una consistencia interna alta. Seguidamente, se hizo un análisis factorial exploratorio (Exploratory Factor Analysis, EFA), mediante el método de factores principales. Por último, se seleccionaron las variables con un peso factorial mayor a 0.4 -de acuerdo a los procedimientos planteados por Joseph F. Hair, William C. Black, Barry J. Babin y Rolph E. Anderson (2013) — para así crear los índices asociados a cada factor. Los índices generados correspondieron a las puntuaciones factoriales obtenidas para cada factor, mediante un promedio ponderado de las variables seleccionadas en cada uno de ellos, y los ponderadores correspondieron al porcentaje de la varianza explicada por cada variable en los factores respectivos. Para establecer los niveles en los índices, se realizó un análisis de clusters no jerárquicos de K-medias que permitió dividir cada índice en cuatro grupos. Así fue posible que la interpretación de los resultados adhiriera a la escala de medición original de las variables.

\section{Resultados y discusión}

\section{Caracterización sociodemográfica de los alumnos}

Uno de los primeros pasos de esta investigación fue identificar los perfiles sociales de los alumnos que asisten a estos liceos públicos de excelencia, por medio de un conjunto de preguntas más específicas y detalladas que las incluidas en la caracterización sociodemográfica disponible en el Ministerio de Educación. Los datos recopilados permiten concluir, en cuanto a la profesión de los progenitores, que un cuarto de los padres $(25,5 \%)$ son empleados administrativos de empresas privadas, públicas o Fuerzas Armadas o técnicos especializados - porcentaje similar al encontrado para las madres (24\%) - . Seguidamente, el $19,7 \%$ de los padres trabaja como obrero calificado, capataz o microempresario. En el caso de las madres, el segundo porcentaje más alto es $22,5 \%$ que corresponde a las que laboran en trabajos ocasionales o informales. En tercer lugar, surgen los trabajadores de carreras técnicas o paramédicas que trabajan en forma dependiente (el $14,6 \%$ de los padres y el $15,8 \%$ de las madres). Las profesiones que exigen más capitales culturales y que se asocian a una posición más privilegiada en la estructura social tienen poca preponderancia: el $11,5 \%$ y el $10,6 \%$ de los padres son, respectivamente, "profesionales con grado de licenciatura trabajando en forma dependiente en empresa privada, pública o Fuerzas Armadas" y "dueño, gerente o subgerente de pequeña y mediana empresa, profesional independiente de carreras tradicionales o directores de establecimientos educacionales". En las 
madres, los porcentajes son bastante más bajos y corresponden, respectivamente, a 7 y 5,3\%. Las ocupaciones asociadas a las élites están prácticamente ausentes — solamente el 1,2\% de los padres y el 0,8\% de las madres son "empresarios o propietarios de una gran empresa, director o alto ejecutivo de la misma"-, datos que comprueban que estos colegios no reciben en porcentajes significativos alumnos de las más altas franjas sociales chilenas.

Sobre la escolaridad de los progenitores, el nivel de enseñanza media completa tiene porcentajes más significativos (29,8\% de los padres y $32,9 \%$ de las madres), seguido de la técnico-profesional completa (respectivamente, 22,6 y $26,8 \%$ ) y la enseñanza universitaria completa (respectivamente, 20,5 y $17,1 \%)$. Se observa un escaso porcentaje de padres y madres con maestría (3,3 y $2,4 \%$ ) y doctorado $(0,8$ y $0,5 \%$ ).

Otro indicador de capital cultural —en el estado objetivado, según la terminología bourdieusiana- es la posesión de libros en la casa. Con respecto a la lectura, y según datos de la Organización de las Naciones Unidas para la Educación, la Ciencia y la Cultura (UNESCO, 2011), Chile se destaca por su elevado consumo de libros e, incluso, es el país de América Latina con menor porcentaje (20\%) de no lectores. En el año 2010, el promedio de libros por hogar era de 46,9 (Cussen, 2010, p. 27). En el caso de estos alumnos, el 33,2\% posee entre 11 a 50 libros no escolares en sus hogares, el 30,2\% más de 100 libros y el 27,6\% entre 51 y 100, lo que indicia la pertenencia a familias con niveles considerables de inversión en la cultura libresca.

En relación con el perfil social de los alumnos, resulta claro que, aunque estos colegios compartan con las escuelas de élite el prestigio y la larga tradición de excelencia académica (Gessaghi \& Méndez, 2015), no comparten con estas el perfil de su público escolar, socialmente homogéneo y constituido por "herederos", es decir, jóvenes provenientes de las familias más dotadas de capital económico-social y, sobre todo, cultural (Bourdieu \& Passeron, 1964). En línea con las conclusiones de otros estudios (Fontaine \& Urzúa, 2014; Valenzuela \& Allende-González, 2012), los datos reflejan una relativa diversidad social en estos liceos públicos, donde las élites y las franjas más vulnerables de la estructura social chilena tienen una presencia muy residual: las primeras porque optan preferencialmente por el entre-soi (Van Zanten, 2009, p. 242) de los colegios particulares pagados; y las segundas, porque las barreras escolares y económicas no les dejan más alternativa que los colegios municipales sin el estatus de liceo emblemático (García-Huidobro \& Bellei, 2003). La gran mayoría de los alumnos encuestados se (auto) identifica no con la élite social, sino con la élite académica, que ha sido, desde la fundación de estos establecimientos, la destinataria de su proyecto republicano y democrático de movilidad social por la vía del mérito, como señalan Arturo Fontaine y Sergio Urzúa (2014).

\section{Representaciones sobre los factores de excelencia}

En cuanto a las percepciones de los alumnos sobre los factores que llevan a la excelencia, a partir de los procedimientos estadísticos explicados anteriormente, ha sido posible identificar cinco índices que reflejan las distintas dimensiones - personales, organizacionales y familiares - con impacto en el éxito, según los encuestados: (1) el background sociodemográfico de la familia y calidad del colegio; (2) el sentimiento de pertenencia al colegio y el apego a los profesores; (3) la competencia y la motivación de escuela, profesores y director; (4) la inteligencia y el esfuerzo; (5) la participación en actividades extraescolares. 
Tabla 2

Variables relativas a factores que permiten alcanzar la excelencia, basados en la pregunta: A tu juicio, ¿qué factores permiten a un alumno alcanzar la excelencia?

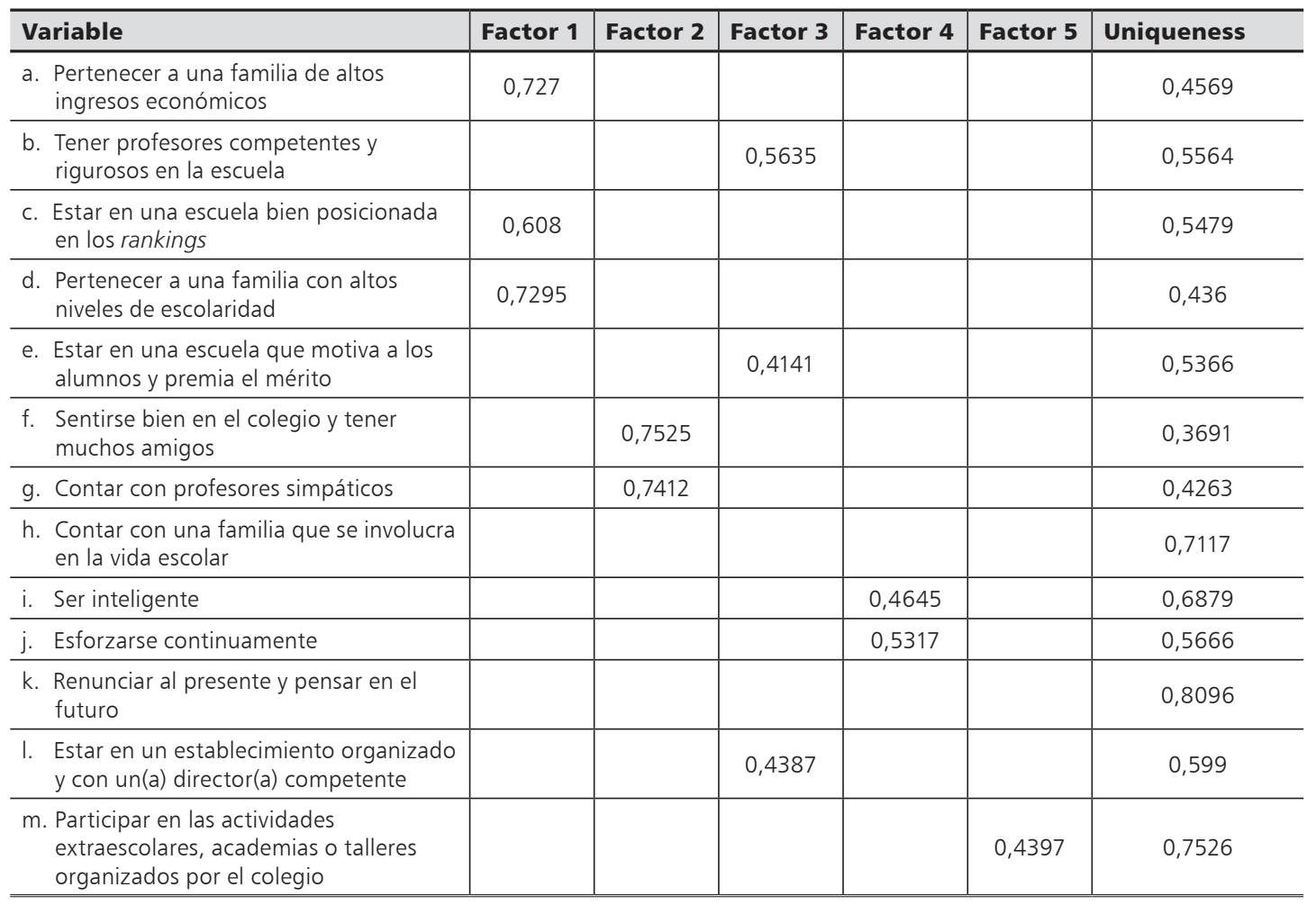

Fuente: elaboración propia

En la tabla 3 se presentan los resultados obtenidos mediante el análisis de cluster realizado sobre los índices que permitió identificar el porcentaje de estudiantes que están de acuerdo o muy de acuerdo con el constructo representado en cada índice.

Tabla 3

Índices estimados, interpretación y porcentaje de alumnos de acuerdo o muy de acuerdo con cada factor de excelencia

\begin{tabular}{|c|c|c|c|}
\hline Tópico & Índices & $\begin{array}{l}\text { Porcentaje } \\
(\%)\end{array}$ & $\begin{array}{l}\text { Número de } \\
\text { observaciones }\end{array}$ \\
\hline \multirow{10}{*}{$\begin{array}{l}\text { Factores que } \\
\text { permiten alcanzar } \\
\text { la excelencia } \\
\text { académica }\end{array}$} & \multirow{2}{*}{ (1) Background sociodemográfico de la familia y calidad del colegio } & 0,64 & \multirow{2}{*}{1.794} \\
\hline & & $(0,48)$ & \\
\hline & \multirow{2}{*}{ (2) Sentimiento de pertenencia al colegio y apego a los profesores } & 0,69 & \multirow{2}{*}{1.806} \\
\hline & & $(0,46)$ & \\
\hline & \multirow{2}{*}{ (3) Competencia y motivación de escuela, profesores y director } & 0,58 & \multirow{2}{*}{1.789} \\
\hline & & $(0,49)$ & \\
\hline & \multirow{2}{*}{ (4) Inteligencia y esfuerzo } & 0,47 & \multirow{2}{*}{1.797} \\
\hline & & $(0,50)$ & \\
\hline & \multirow{2}{*}{ (5) Participación en actividades extraescolares } & 0,52 & \multirow{2}{*}{1.805} \\
\hline & & $(0,50)$ & \\
\hline
\end{tabular}

Fuente: elaboración propia 
Al analizar la tabla 3, se observa que, para los alumnos, el principal factor de excelencia es el sentimiento de pertenencia al colegio y el apego a los profesores (69\%), rasgos también muy valorados por los alumnos durante las entrevistas. La identidad de alumno de excelencia se crea y consolida a partir del sentido de pertenencia a un colegio que alía a la larga tradición de excelencia las dimensiones afectivas, reforzando en cada uno las disposiciones y los valores compartidos y la voluntad de contribuir a la defensa de la imagen de marca de estos colegios, como explica una entrevistada:

Es como parte de una identidad, entonces uno asume cierta identidad como mi colegio es tan bacán [bueno], aquí somos tan amigos... No sé, entonces como que uno entra en esa onda y en esa identidad más como en grupo, entonces vamos todas ah, somos bacanes. Viene otra persona de otro colegio y "soy de un emblemático" (e4, femenino, Liceo 3, $3^{\circ}$ Medio).

También reiteradas como eficaces para el desempeño académico son las competencias relacionales y emocionales (Barrère, 2002) de sus profesores, considerados "demasiado humanos" (e2, femenino, Liceo 2, $3^{\circ}$ Medio) y atentos a la persona que existe en cada alumno:

Ahí como que hay empatía de las dos partes, porque igual siempre cuando un profe, a mí me ha pasado, un profe te ve mal tampoco va y así como qué le pasa, por qué... (e3, femenino, Liceo 2, $3^{\circ}$ Medio).

Como que pregunta si uno tiene algún problema, si te ayuda, quieres salir un rato... (e2, femenino, Liceo $2,3^{\circ}$ Medio).

Estas dimensiones de índole expresiva se alejan de las que tradicionalmente se asocian a la excelencia en contextos educativos orientados hacia los resultados académicos (Quaresma, 2015), pues se priorizan los factores de naturaleza meritocrática e individualista que, según la narrativa de la performatividad (Ball, 2012), confieren a cada individuo "la responsabilidad por trabajar más, más rápido y mejor" (p. 31). De hecho, el factor inteligencia y esfuerzo no es el más preponderante en las respuestas de los encuestados. La mitad de los alumnos (47\%) adhiere a los valores de esa ficción meritocrática que consiste en creer o hacer creer que "los logros son esencialmente basados en sus propios talentos o esfuerzos" (Van Zanten, 2015 , p. 36). Sin embargo, en las entrevistas es más expresiva la valoración del esfuerzo - no de la inteligencia-como predictor de excelencia, lo que se puede interpretar por el hecho de que estos estudiantes están insertos en una sociedad que tiene "el mérito como principio de justicia" (Araujo \& Martuccelli, 2015, p. 1505) y que cree en la escuela como ascensor social.

Bastante más expresivo es el porcentaje de alumnos (64\%) que asocian a la excelencia factores relacionados con la pertenencia sociocultural de la familia (Bourdieu \& Passeron, 1964) y con la posición del colegio en los rankings escolares, como nos dice uno de los entrevistados:

Yo creo que es muy importante también (...) el tema yo creo de la realidad social en la que uno viva, social y económica también. También afecta, yo creo, el tema familiar yo creo que sí afecta, quizá no en gran medida pero tiene algún... Tiene ahí algo que afecta. Y también yo creo que el colegio, la realidad de la calidad como establecimiento o, como hablábamos de la excelencia académica del establecimiento, yo creo que va en... A ver, como en el día a día del colegio (e5, masculino, Liceo 1, 4 Medio). 
Cabe señalar que aunque la variable "contar con una familia que se involucre en la vida escolar" no se integre en los índices estimados, esta es considerada importante o muy importante por $81 \%$ de los alumnos; este resultado va en el mismo sentido de los hallazgos de la literatura que apuntan las dinámicas internas de las familias como factores predictores de la excelencia (Lahire, 2008; Nogueira, 2004). Como refiere el mismo alumno:

Yo creo que es fundamental [la familia] porque al fin y al cabo uno tiene el respaldo por lo menos yo siento el respaldo de mi familia que independiente de si no logro altiro [de inmediato] el éxito, ellos van a estar ahí, apoyándome y ayudándome a conseguirlo (e5, masculino, Liceo 1, $4^{\circ}$ Medio).

Seguidamente, y con un porcentaje significativo (58\%), surge el índice "competencia y motivación por parte de la escuela, directores y profesores", que señala la importancia de contar con profesores competentes y rigurosos, estar en una escuela que motiva y premia el mérito y estar en un establecimiento organizado y con un(a) director(a) competente. Dado que estos son alumnos que no pertenecen a las clases sociales para las cuales el éxito escolar es naturalizado y que depositan en estos colegios una gran parte de sus expectativas de una trayectoria escolar exitosa, no sorprende que los profesores y los directores sean considerados un factor importante de excelencia. Como refiere uno de los alumnos, el factor fundamental para lograr la excelencia es "el profesor, la disposición del profesor, la calidad del profesor, si te motiva, y el contenido en sí, si te lo pasan bien, te lo enseñan bien, y si te ayudan a enfrentar los desafíos que un estudiante tiene que enfrentar: Ia PSU y posteriormente" (e1, masculino, Liceo 6, $4^{\circ}$ Medio). Otra alumna agrega:

Yo noto mucha diferencia. He visto que los profes que están acá vienen de universidades muy buenas y tienen muy buen currículum pero están acá y tienen mucha, mucha, mucha vocación porque hay otros colegios, tengo amigas que están en colegios privados que pagan mucha plata pero pasan la materia, pruebas y ya está. Acá nuestros profes se toman las dos horas para revisar pregunta por pregunta, en lenguaje por ejemplo, es espectacular. (...) Es muy buena, hay de verdad un aprendizaje (e4, femenino, Liceo 3, $4^{\circ}$ Medio).

Por otro lado, la referencia al rol del director viene en línea con los estudios que señalan su contribución en la construcción de la excelencia por un "efecto-indirecto: no es él quien trabaja en las salas de clases, pero puede contribuir para la creación de condiciones para que se trabaje bien en estas" (Bolívar, 2012, p. 57).

Finalmente, es de subrayar la percepción sobre la contribución positiva de las actividades extraescolares (talleres, academias, etc.) para alcanzar la excelencia, que es compartida por el $52 \%$ de los encuestados. De hecho, la literatura ha planteado la importancia de los aprendizajes formales e informales en la socialización de los alumnos y en sus trayectorias escolares (Rogers, 2014), la cual es documentada por los profesores, quienes hablan de ventajas en términos del desempeño escolar y también de aptitudes (Gaussel, 2013). Así se explica la obsesión de los padres para garantizar un valor escolar a los tiempos libres de sus hijos, retirándoles incluso el necesario derecho al descanso y al sueño, como denuncia Dominique Glasman (2005). Las clases medias son las que más invierten en actividades extraescolares para los hijos, según Annette Lareau (2011). Movilizadas por el éxito escolar de los niños, ellas conciben las actividades de "ocio cultivado" como un indiscutible valor agregado escolar, en comparación con el "crecimiento natural" propiciado a los hijos por las clases populares.

En cuanto a las motivaciones personales para ser exitoso, obtenidas por medio de análisis estadísticos descriptivos, contrariamente a lo que se podría esperar, y aunque estos colegios sean la vía de ingreso a las mejores carreras y universidades (Fontaine \& Urzúa, 2014), los jóvenes defienden, principalmente, razones no instrumentales. Así, para la casi totalidad de los alumnos (92,8\%), lograr la excelencia es importante para sentirse bien con el logro. La incorporación del estatuto de excelencia en la identidad de los alumnos (Vieira, 2014) lleva a que la excelencia empiece a ser percibida como una condición sine qua non del bienestar individual, lo que conduce a una continua y desafiante "competencia con uno mismo" (e1, masculino, Liceo 6, $4^{\circ}$ Medio), no tanto en el sentido de no defraudar las expectativas de los demás sino en el sentido de no defraudar sus expectativas personales. La "carrera de obstáculos" que significa el camino hacia la excelencia (Perrenoud, 1995) no se vence sin la fuerte motivación, persistencia, perfeccionismo y definición de metas que la literatura les atribuye como rasgos diferenciadores (Álvarez-Rojo, García-Jiménez, Gil-Flores \& Romero-Rodríguez, 1999; Palhares \& Torres, 2011; Vieira, 2014) y que son también referidos por los alumnos durante las entrevistas. Como nos dicen los estudiantes, el logro de la excelencia depende "de la perseverancia de cada uno" (e5, masculino, Liceo $6,3^{\circ}$ Medio), pero también de la "responsabilidad y autoexigencia" (e4, femenino, Liceo 2, $4^{\circ}$ Medio) y del "interés y motivación" (e1, femenino, Liceo 2, $3^{\circ}$ Medio). Como explica otro alumno: 
Yo pienso que para ser un alumno de excelencia en cualquier ámbito que uno quiera la excelencia, hay que fijarse metas y hay que formar una determinación (e3, masculino, Liceo 1, $3^{\circ}$ Medio).

Sin embargo, hay que registrar el alto porcentaje $(69,8 \%)$ de encuestados que va más allá de esta conceptualización individualista de excelencia que la concibe como un fin en sí mismo y para sí mismo, defendiendo que ser un alumno de excelencia es importante para ser un ciudadano atento y participativo:

Para mí, excelencia académica es el tipo de educación donde te forman no solo en el contenido del saber y responder, sino en valores, responsabilidad, civilidad y eso, deber... algo completo (e1, masculino, Liceo $6,4^{\circ}$ Medio).

Para comprender la centralidad de esta dimensión, importa tener en consideración que estamos ante alumnos socializados en colegios que tienen como principio el ideal de formar ciudadanos involucrados en la vida social, política y cívica, como resulta claro de la lectura de los proyectos educativos. Además, no sorprende el protagonismo de los alumnos de estos colegios en lo que algunos investigadores consideran ser el movimiento social de mayor relevancia de la última década (Mönckeberg, 2011): las luchas estudiantiles que quedaron conocidas como "revolución de los pingüinos" y que condujeron a la "reestructuración institucional del sistema escolar más significativa desde la vuelta a la democracia, solo comparable con la reforma neoliberal de principios de los años 80" (Bellei, Contreras \& Valenzuela, 2010, p. 11). Tomas, paros y manifestaciones grandiosas marcaron la agenda de protesta de estos estudiantes contra el segregacionismo y el elitismo de un sistema escolar que proporciona vías de excelencia para los más destacados y condena a los demás a una educación pública sin calidad, y pusieron en el centro del debate el derecho a un servicio público de educación gratuito y de excelencia para todos y la necesidad de que esta no se disocie jamás de la equidad (Duru-Bellat \& Mingat, 2015).

\section{Prácticas de estudio de los alumnos de excelencia}

Al considerar que la "producción de la excelencia" requiere un componente de dedicación al trabajo escolar por parte de los alumnos, incluso por parte de los herederos (Nogueira, 2004; Vieira, 2014), importa conocer las prácticas de estudio de estos jóvenes. En primer lugar, los niveles educativos que ellos se proponen

1 Marchas y protestas que se iniciaron en 2006 en defensa de la educación pública gratuita y de calidad, protagonizadas por los estudiantes de educación secundaria y superior. alcanzar son elevados, bastante más que los completados por sus padres. Así, el 31,7\% quiere completar una carrera universitaria de pregrado y el $25,8 \%$ desea obtener un doctorado. El 19,8 y el 19,3\% tienen por meta, respectivamente, un magíster y un doctorado. No sorprende que para lograr estas metas los alumnos se involucren en su quehacer escolar cotidiano, cumpliendo con diligencia su "oficio de alumno" (Perrenoud, 1995). Así, el 81,2\% de los alumnos dice asistir siempre y puntualmente a clases, el 73,6\% de los alumnos refiere intentar hacer lo mejor posible las tareas escolares y el 77,6\% dice cumplir casi siempre o siempre las demandas de los profesores.

Uno de los desafíos que hay que enfrentar bajo un régimen de "alta competencia" escolar se relaciona con el tiempo y con su gestión (Darmon, 2015), como estos estudiantes también reconocen durante las entrevistas, cuando hablan "del tiempo que se pasa volando" (e1, masculino, Liceo 6, $3^{\circ}$ Medio), de la necesidad de "aprovechar los tiempos cortos" (e3, femenino, Liceo2, $4^{\circ}$ Medio) para estudiar, como los trayectos en metro, o de definir rutinas de trabajo.

En el caso de estos alumnos, sabemos que el número de horas que dedican al estudio fuera del colegio es superior al promedio de horas destinado por los alumnos chilenos a esa tarea, que no alcanza las 4 por semana, según los indicadores de la Organización para la Cooperación y el Desarrollo Económicos (OCDE, 2014). El 49,1\% de los encuestados estudia entre 1 y 3 horas por día durante la semana y el 30,5\% menos de 1 hora por día. Durante el fin de semana, los valores no presentan diferencias significativas atingiendo, respectivamente, el 42,3 y 36,1\%. Solamente el $5 \%$ de los alumnos declara estudiar más de 5 horas diarias, lo que casi supone poner "su vida entre paréntesis" (Darmon, 2015, p. 85) en su calidad de jóvenes. Así, los casos de alumnos que tienen riesgos reales de sufrir las "enfermedades de la excelencia" que suelen acompañar la "persecución demasiado absoluta de una excelencia y de una performance siempre mayor" son escasos (Aubert \& De Gaulejac, 1991, p. 315).

En lo que se refiere al acompañamiento de las tareas escolares por parte de los padres, que la literatura correlaciona positivamente con el éxito escolar (Lahire, 2008), la encuesta revela que en su mayoría, los alumnos estudian solos (el 94,8\% lo hace siempre o casi siempre). La presencia de los padres en el momento de la preparación de clases y pruebas es escasa y queda detrás, incluso, del acompañamiento del profesor o de los hermanos. El 69,6\% nunca estudia con sus padres y solamente el $2,4 \%$ estudia siempre o casi siempre con ellos. Cuando tienen alguna duda en el estudio, un gran porcentaje de alumnos no cuenta nunca con el apoyo de sus padres (47,1\%). Solamente el $2,6 \%$ de los alumnos consulta a sus padres en caso de necesidad 
de aclaración adicional. La "invisibilidad" de los padres en la realización de las tareas escolares puede ser reflejo de una desmovilización resultante de su handicap escolar para auxiliar a los hijos, como es muy común en las familias con menos capitales culturales, en las que con cierta frecuencia son los hermanos mayores los que se ocupan de esa misión (Lahire, 2008). La referencia de los encuestados al acompañamiento realizado por la fratría da robustez a la hipótesis de que algunos de los encuestados integran configuraciones familiares de este tipo. No obstante, la falta de acompañamiento puede también reflejar las dificultades de conciliación de los tiempos consagrados a la vida familiar y profesional, agravada por la inserción de las mujeres en el mundo laboral —esta hipótesis puede ser considerada a pesar de la escasa presencia de las mujeres chilenas en el mercado laboral, que permanece una de las más bajas en América Latina y países de la OCDE (INE, 2015) - . La "invisibilidad" de los padres puede, sin embargo, ser reflejo también de un modelo de educación familiar para la autonomía, muy frecuente en las clases medias (Lareau, 2011) y muy valorado en el campo escolar (Lahire, 2008), que a la edad de estos alumnos estaría ya dando frutos. Ante hijos autónomos, los padres estarían tranquilos en cuanto al cumplimiento de las tareas escolares. Es posible admitir que la confianza de estos padres es no solamente en la destreza académica y en la responsabilidad de los hijos, sino también en la calidad de los colegios a que ellos asisten y de los profesores que los acompañan, lo cual lleva a estos padres a no sentir la necesidad de una monitorización intensa del trabajo escolar de sus hijos. De hecho, varios estudios señalan la existencia, por parte de los padres, de un ajustamiento del tiempo y atención dedicados al apoyo escolar en función de la percepción que ellos tienen del desempeño académico de sus hijos (Hoover-Dempsey, Battiato, Walker, Reed, DeJong \& Jones, 2001; Marcotte, Fortin, Cloutier, Royer \& Marcotte, 2005). Como refiere un alumno:

Yo les doy la confianza [a los padres] de que yo me esfuerzo y si me saco un 3 yo voy a encargarme de subirlo. Igual se relajan un poco, descansan en mí, sabiendo que en el fondo somos gente responsable en el liceo entonces para mis papás, por lo menos, es un relajo saber que yo les doy la tranquilidad que no tienen que estar encima de mí, cargándome (e1, masculino, Liceo 5, $3^{\circ}$ Medio).

En caso de necesidad de aclaración adicional de dudas, los compañeros de colegio funcionan como red de apoyo. Más de la mitad de los jóvenes encuestados $(61,9 \%)$ dice contar siempre o casi siempre con el apoyo escolar de sus pares. Además, el 23,6\% declara estudiar siempre o casi siempre con ellos. El apoyo de los amigos — claramente superior al de los padres - revela la existencia de relaciones de amistad, solidaridad y ayuda recíproca que cuestionan la idea de un clima de exacerbado individualismo y competencia generador de tensiones y rivalidades entre los alumnos, en lucha por el acceso a la mejor nota o al estatus de mejor alumno de la clase (Perrenoud, 1995):

Si hay una pregunta que se sabe que va a entrar en la prueba, uno también la comparte, no es como que se la deje para uno, porque igual sería triste e individualista, poh (e2, masculino, Liceo 1, $3^{\circ}$ Medio).

Sin embargo, el soporte más utilizado por estos alumnos es internet, lo que está en línea con los estudios que señalan la amplia difusión de las tecnologías de información y comunicación entre los adolescentes, que las 
ocupan no solamente para fines lúdicos, sino también para el trabajo escolar (Burban, Cottier \& Michaut, 2013). Las tradicionales pesquisas en la biblioteca están actualmente siendo reemplazadas por los motores de búsqueda y Wikipedia (Donnat, 2009). Chile, a pesar de su fuerte "brecha tecnológica", ha registrado en el último decenio un fuerte aumento en términos de acceso y uso de las nuevas tecnologías de información; en el año 2010 , en el $68,9 \%$ de los hogares de la región metropolitana había un computador (Agostini \& Willington, s.f.) En consecuencia, es un resultado esperado que el $81,2 \%$ de los alumnos refiera consultar las páginas web, cuya influencia suplantó ya claramente el peso del "libro" en casos de duda. La mitad de los alumnos $(50,5 \%)$ solamente consulta los libros en caso de dudas "algunas veces" y el 7,1\% lo hace siempre.

El rol de los profesores - aunque aparezca claramente detrás del papel de internet y de los compañeros de colegio- es importante como apoyo a los alumnos, incluso bastante más preponderante que el peso que tiene la familia. El 33,7\% de los alumnos dice siempre o casi siempre contar con los profesores en caso de duda. Como dice un entrevistado:

Si alguien tiene alguna duda, lo bueno es que hay profesores que la pueden responder sin ningún problema (e3, masculino, Liceo 6, $3^{\circ}$ Medio).

\section{Conclusión}

El análisis presentado ha revelado que las perspectivas de los alumnos de liceos públicos de alto rendimiento sobre los factores de excelencia se caracterizan, esencialmente, por dimensiones no instrumentales, al contrario de lo que sería esperable en contextos sociales y escolares marcados por las lógicas de competencia y mercantilización, como es el caso de Chile. Para estos jóvenes exitosos que han superado las barreras académicas de acceso a colegios altamente selectivos, el principal factor de excelencia es el sentimiento de pertenencia a la escuela y el apego a los profesores, lo que significa que la excelencia se potencia esencialmente en contextos educativos donde el alumno se sienta acogido, comparta lazos de amistad con los compañeros y cuente con profesores simpáticos. Las dimensiones que la literatura refiere como los mejores predictores del éxito para los vencedores de la competencia escolar - la inteligencia y el esfuerzo- no asumen la preponderancia esperada, cuando se analizan estadísticamente como un único factor. De hecho, el background sociodemográfico de la familia y la calidad del colegio se consideran un predictor más decisivo del éxito que el factor individual "inteligencia y esfuerzo" - estos resultados son sorprendentes, si tenemos en consideración que, como refiere Élise
Tenret (2008), los mejores alumnos son los que valoran menos el peso de la pertenencia social y naturalizan su éxito por medio del don y del mérito. Una posible explicación de la importancia atribuida al factor contextual (sea familiar o escolar) —y que encuentra eco en la percepción ampliamente compartida por los estudiantes de que la excelencia es importante para ser un ciudadano atento y participativo - reside en la activa participación de estos alumnos en luchas y movimientos sociales de rechazo a la mercantilización y competencia en el sistema educativo y en la defensa de la igualdad de oportunidades y calidad de la escuela pública chilena. Más aún si tenemos en consideración que los datos fueron recopilados en el año 2015, durante un período de fuerte protesta estudiantil. Por otro lado, el hecho de que estos alumnos estén insertos en una sociedad con importantes indicadores de desigualdad -en términos sociales, económicos, educativos y residenciales - en los cuales la idea de reproducción social está muy instalada, podrá llevarlos a considerar importante la pertenencia social. Otra explicación puede ser encontrada en la pertenencia social de estos alumnos: al no integrar ni la élite social, ni el grupo de los más vulnerables, estos jóvenes de clase media tienen conciencia de la importancia del entorno familiar y escolar para lograr la anhelada movilidad social ascendente.

Otro resultado no esperado se relaciona con la invisibilidad de indicios de una relación tensada por las demandas de excelencia. Por un lado, los factores que los alumnos asocian al éxito no tienen cariz de negatividad (la idea de trabajo arduo presente en el concepto de esfuerzo no es una dimensión central); por otro lado, los datos indican que los estudiantes gestionan de manera eficaz su tiempo de estudio. Así, aunque el número de horas de estudio al día supere el promedio chileno, pocos alumnos estudian, durante la semana o el fin de semana, más de cinco horas al día.

Estos datos abren un conjunto de interrogantes que requieren una reflexión más profundizada y alargada que involucre la escucha activa de otros agentes escolares, como los padres, profesores y directivos. Además, la representatividad de este estudio ganaría si los estudiantes de los demás liceos emblemáticos fuesen encuestados, para garantizar que estos resultados no representan solamente a un subgrupo de alumnos. Otro aspecto a tener en consideración -y que constituye otra debilidad de esta investigación- es la percepción del resto de los estudiantes del sistema público y privado nacional sobre las temáticas abordadas en este estudio. Solamente la comparación entre las múltiples realidades escolares permite concluir, con más sistematicidad, sobre la especificidad de este grupo de estudiantes "emblemáticos". De hecho, un estudio más macro permitiría (re)interpretar los sentidos 
que los jóvenes atribuyen a los factores que potencian la excelencia y, a la vez, conocer las prácticas que constituyen el diario escolar y extraescolar de jóvenes insertos en un contexto escolar fuertemente segregativo y direccionado hacia la competencia y al éxito académico medido con pruebas estandarizadas. Reflexiones y debates que quedan pendientes para nuevos estudios sociológicos.

\section{Sobre la autora}

Maria Luísa Quaresma es doctorada en sociología por la Universidade do Porto (Portugal). Es profesora e investigadora en el Instituto de Estudios Sociales y Humanísticos de la Facultad de Ciencias Sociales y Humanidades (Universidad Autónoma de Chile) e investigadora en el Instituto de Sociología de la Universidade do Porto (Portugal).

\section{Referencias}

Agostini, Claudio A. \& Willington, Manuel (s.f.). Encuesta sobre acceso, uso y usuarios de internet banda ancha en Chile. Santiago: Observatorio Social de la Universidad Alberto Hurtado. Disponible en: http://www. uai.cl/RePEc/uai/wpaper/wp_016.pdf

Álvarez-Rojo, Víctor; García-Jiménez, Eduardo; Gil-Flores, Javier \& RomeroRodríguez, Soledad (1999). Exigencias del trabajo universitario desde la perspectiva de los alumnos. Revista de Investigación Educativa, 17 (2), 385-392. Disponible en: http://revistas.um.es/rie/article/down load/121941/114621

Amatea, Ellen S.; Smith-Adcock, Sondra \& Villares, Elizabeth (2006). From Family Deficit to Family Strength: Viewing Families Contributions to Children's Learning from a Family Resilience Perspective. Professional School Counseling, 9 (3), 177-189. Disponible en: http://professionals choolcounseling.org/doi/pdf/10.5330/prsc.9.3.43751461038m4m68

Araujo, Kathya \& Martuccelli, Danilo (2015). La escuela y la cuestión del mérito: reflexiones desde la experiencia chilena. Educação \& Pesquisa, 41 (número especial), 1503-1518. Disponible en: http://www.re vistas.usp.br/ep/article/viewFile/109911/108410

Assaél-Budnik, Jenny; Cornejo-Chávez, Rodrigo; González-López, Juan; Redondo-Rojo, Jesús; Sánchez-Edmonson, Rodrigo \& SobarzoMorales, Mario (2011). La empresa educativa chilena. Educação \& Sociedade, 32 (115), 305-322. Disponible en: http://www.scielo.br/ pdf/es/v32n115/v32n115a04.pdf

Aubert, Nicole \& Gaulejac, Vincent de (1991). Le coût de l'excellence. Paris: Éditions Seuil.

Ball, Stephen J. (2012). Global Education Inc.: New Policy Networks and the Neo-liberal Imaginary. New York: Routledge.

Bellei, Cristián (2015). El gran experimento. Mercado y privatización de la educación chilena. Santiago de Chile: LOM Ediciones.

Bellei, Cristián; Contreras, Daniel \& Valenzuela, Juan Pablo (2010). Viejos dilemas y nuevas propuestas en la política educacional chilena. En Cristián Bellei, Daniel Contreras \& Juan Pablo Valenzuela (eds.). Ecos de la Revolución Pingüina. Avances, desafíos y silencios en la reforma educacional, 11-27. Santiago: Pehuén. Disponible en: http://unicef. cl/web/ecos-de-la-revolucion-pinguina-avances-debates-y-silenciosen-la-reforma-educacional/

Bellei, Cristián; Valenzuela, Juan Pablo; Vanni, Xavier \& Contreras, Daniel (2014). Las claves del mejoramiento escolar sostenido. En Cristián 
Bellei, Juan Pablo Valenzuela, Xavier Vanni \& Daniel Contreras (eds.). Lo aprendí en la escuela ¿Cómo se logran procesos de mejoramiento escolar?, 55-94. Santiago: LOM. Disponible en: http:// unicef.cl/web/wp-content/uploads/2016/04/Loaprendi---en-la-escuela.pdf

Bolívar, Antonio (2012). Melhorar os processos e os resultados educativos. O que nos ensina a investigação. Vila Nova de Gaia: Fundação Manuel Leão.

Bourdieu, Pierre \& Passeron, Jean-Claude (1964). Les héritiers. Les étudiants et la culture. Paris: Les Editions de Minuit.

Bressoux, Pascal (2012). L'influence des pratiques enseignantes sur les acquisitions scolaires des élèves. En Regards Croisés sur l'Économie, volumen 2 (12), L'école, une utopie à reconstruiré, 207-218. Paris: La Découverte. Disponible en: https://www.cairn.info/revue-regards-croisessur-I-economie-2012-2.htm

Brunner, José Joaquín \& Elacqua, Gregory (2003). Informe Capital humano en Chile. Santiago de Chile: Universidad Adolfo Ibáñez, Escuela de Gobierno. Disponible en: www.oei.es/historico/etp/ informe_capital_humano_chile_brunner.pdf

Bruns, Barbara \& Luque, Javier (2014). Professores Excelentes. Como melhorar a aprendizagem dos estudantes na América Latina e no Caribe. Washington: Grupo Banco Mundial. Disponible en: http://www.geografia.fflch.usp.br/gradua cao/apoio/Apoio/Apoio_Gloria/1s2017/Banco_ mundial_e_o_ensino_na_america_latina.pdf

Burban, François; Cottier, Philippe \& Michaut, Christophe (2013). Les usages numériques des lycéens affectent-ils leur temps de travail personnel? Revue STICEF, 20, 1-28. Disponible en: http://sticef. univ-lemans.fr/num/vol2013/05-burban-cren/ sticef_2013_NS_burban_05p.pdf

Castets-Fontaine, Benjamin (2011). Le cercle vertueux de la réussite scolaire. Le cas des élèves de Grandes Écoles issus de "milieux populaires". Paris: Éditions Modulaires Européennes.

Chile, Instituto Nacional de Estadísticas, INE (2015). Mujeres en Chile y mercado del trabajo. Participación laboral femenina y brechas salariales. Santiago de Chile: Instituto Nacional de Estadísticas, INE. Disponible em: http://nuevoportal.ine. cl/docs/default-source/laborales/ene/publicacio nes/mujeres-en-chile-y-mercado-del-trabajo--participaci\%C3\%B3n-laboral-femenina-y-bre chas-salariales.pdf?sfvrsn $=4$

Cussen, Albert (dir.) (2010). Chile y los libros 2010. Santiago: Fundación La Fuente/Adimark GFK. Disponible en: http://www.fundacionlafuente. cl/wp-content/uploads/2010/11/Chile-y-los-
libros-2010_FINAL-liviano.pdf, http://www.fun dacionlafuente.cl/documentos/

Darling-Hammond, Linda (2000). How Teacher Education Matters. Journal of Teacher Education, 51 (3), 166-173. Disponible en: http://journals.sagepub. com/doi/pdf/10.1177/0022487100051003002

Darmon, Muriel (2015). Classes préparatoires. La fabrique d'une jeunesse dominante. Paris: Éditions La Découverte.

Donnat, Olivier (2009). Les pratiques culturelles des français à l'ère numérique: Enquête 2008. Paris: Éditions La Découverte, Ministère de la Culture et de la Communication.

Duru-Bellat, Marie (2010). L'école et les ambivalences du mérite. Revista Portuguesa de Pedagogía, Edición especial 30 Años, 25-32. Disponible en: http://impactum-journals.uc.pt/rppedagogia/ar ticle/download/1349/797/

Duru-Bellat, Marie \& Mingat, Alain (2011). Measuring Excellence and Equity in Education, Conceptual and Methodological Issues. En Kris van den Branden, Piet van Avermaet \& Mieke van Houtte (eds.). Equity and Excellence in Education: Towards Maximal Learning Opportunities for All Students, 21-38. New York: Routledge.

Fontaine, Arturo \& Urzúa, Sergio (2014). El fin de los liceos emblemáticos: ¿Por qué? ¿Cómo? ¿Impacto? Santiago de Chile: Centro Latinoamericano de Políticas Económicas y Sociales de la Pontificia Universidad Católica de Chile, CLAPES UC, Documento de trabajo 6. Disponible en: http://www. clapesuc.cl/assets/uploads/2016/02/educacionsurzua-y-otros-doc-t-6-sept-20141.pdf

García-Huidobro, Juan Eduardo \& Bellei, Cristián (2003). Desigualdad educativa en Chile. Santiago: Universidad Alberto Hurtado, Escuela de Educación.

Gaussel, Marie (2013). Aux frontières de l'École ou la pluralité des temps éducatifs. Dossier d'actualité Veille et Analyses, 81, 1-20. Disponible en: http:// ife.ens-lyon.fr/vst/DA-Veille/81-janvier-2013.pdf

Gessaghi, Victoria \& Méndez, Alicia (2015). Elite Families and Schools in Buenos Aires. The Role of Tradition and School Social Networks in the Production and Reproduction of Privilege. En Agnés van Zanten, Stephen J. Ball \& Brigitte Darchy-Koechlin (eds.). World Yearbook of Education 2015. Elites, Privilege and Excellence. The National and Global Redefinition of Educational Advantage. New York: Routledge.

Glasman, Dominique (2005). Leur reste-t-il du temps pour jouer? Diversité: Ville école Intégration, 141, 51-57.

Gubbins-Foxley, Veronica (2014). Estrategias educativas de familias de clase alta. Un estudio exploratorio. 
Revista Mexicana de Investigación Educativa. RMIE, 19 (63), 10691089. Disponible en: http://www.redalyc.org/pdf/140/14032016 004.pdf

Hair, Joseph F.; Black, William C.; Babin, Barry J. \& Anderson, Rolph E. (2013). Multivariate Data Analysis. London: Pearson Publisher.

Henri-Panabière, Gaële (2010). Des "héritiers" en échec scolaire. Paris: La Dispute.

Hoover-Dempsey, Kathleen; Battiato, Angela C.; Walker, Joan M. T.; Reed, Richard P.; DeJong, Jennifer M. \& Jones, Kathleen P. (2001). Parental Involvement in Homework. Educational Psychologist, 36 (3), 195209. http://dx.doi.org/10.1207/S15326985EP3603_5

Kellerhals, Jean \& Montandon, Cléopâtre (1991). Les stratégies éducatives des familles: milieu social, dynamique familiale et éducation des préadolescents. Lausanne: Delachaux et Niestlé.

Khan, Shamus Rahman (2011). Privilege: The Making of an Adolescent Elite at St. Paul's School. Princeton: Princeton University Press.

Lahire, Bernard (2008). Sucesso escolar nos meios populares. As razões do improvável. São Paulo: Editora Ática.

Lareau, Annette (2011). Unequal Childhoods: Class, Race, and Family Life. Berkeley, Los Angeles, California: University of California Press.

Marcotte, Julie; Fortin, Laurier; Cloutier, Richard; Royer, Égide \& Marcotte, Diane (2005). Evolution de l'engagement parental auprès des élèves en difficulté de comportement et des élèves ordinaires au début du secondaire. Nouveaux Cahiers de la Recherche en Education, 8 (2), 47-56. Disponible en: https://www.erudit.org/fr/revues/ncre/2005v8-n2-ncre0753/1017528ar.pdf

Maroy, Christian (2012). Les politiques d'accountability au service de la confiance dans l'institution scolaire et les enseignants? Revue Suisse des Sciences de l'Éducation, 34 (1), 57-70. Disponible en: http://www.pe docs.de/volltexte/2015/10040/pdf/SZBW_2012_1_Maroy_Les_poli tiques_daccountability.pdf

Martín-Criado, Enrique; Gómez-Bueno, Carmuca; Fernández-Palomares, Francisco \& Rodríguez-Monge, Ángel (2000). Familia de clase obrera y escuela. Bilbao: Editorial Iralka.

Mönckeberg-Pardo, María Olivia (2011). El negocio de las universidades en Chile. Santiago: Debate.

Nogueira, Maria Alice (2004). Favorecimento econômico e a excelência escolar: um mito em questão. Revista Brasileira de Educação, 26, 133144. Disponible en: http://www.scielo.br/pdf/rbedu/n26/n26a10.pdf

Organización de las Naciones Unidas para la Educación, la Ciencia y la Cultura, UNESCO (2011). Comportamiento lector y hábitos de lectura. Una comparación de resultados en algunos países de América Latina. Bogotá: Centro Regional para el Fomento del Libro en América Latina y el Caribe, CERLALC. Disponible en: http://www.cerlalc.org/files/ tabinterno/33c91d_Comportamiento_Lector.pdf

Organización para la Cooperación y el Desarrollo Económicos, OCDE (2014). Panorama de la educación 2014. Madrid: Fundación SantiIlana. Disponible en: http://www.oecd-ilibrary.org/education/educa tion-at-a-glance-2014_eag-2014-en

Palhares, José Augusto \& Torres, Leonor Lima (2011). Percursos de Excelência no Ensino Secundário: representações e práticas de alunos distinguidos num quadro de excelencia. En // Encontro de Sociologia da Educação, Universidade do Porto, 27 e 28 de Janeiro de 2011, 420-438. Porto: Faculdade de Letras. Disponible en: https://repositorium.sdum.uminho. 
pt/bitstream/1822/14186/1/jpalhares\%26leonot_ SocEd2_Porto2011_392-601.pdf

Perrenoud, Philippe (1995). Ofício de aluno e sentido do trabalho escolar. Porto: Porto Editora.

Quaresma, Maria Luísa (2013). Socialización familiar y formación holística: análisis de las prácticas educativas de las clases dominantes. Revista de la Asociación de Sociología de la Educación, 6 (3), 491-503. Disponible en: https://ojs.uv.es/index. php/RASE/article/view/8674/8217

Quaresma, Maria Luísa (2014). Entre o herdado, o vivido e o projetado: Estudo de caso sobre o sucesso educativo em dois colégios privados frequentados pelas classes dominantes. Porto: Edições Afrontamento.

Quaresma, Maria Luísa (2015). Entre a entrega e a renúncia: excelência académica em escolas públicas chilenas de alta performance. Educação e Pesquisa, 41 (número especial), 1487-1501. Disponible en: http://www.scielo.br/pdf/ep/ v41nspe/1517-9702-ep-41-spe-1487.pdf

Rogers, Alan (2014). The Classroom and the Everyday: The Importance of Informal Learning for Formal Learning. Investigar em Educação, // Serie (1), 7-34. Disponible en: http://pages.ie.uminho.pt/ inved/index.php/ie/article/view/3/3

Schneider, Barbara; Keesler, Venessa \& Morlock, Larissa (2010). Les influences familiales sur l'apprentissage et la socialisation des enfants. En Hanna Dumont, David Istance \& Francisco Benavides (eds.). Comment apprend-on? La recherche au service de la pratique, 265-299. Paris: OCDE. Disponible en: http://www.keepeek.com/Digital-Asset-Mana gement/oecd/education/comment-apprend-on/ les-influences-familiales-sur-l-apprentissage-etla-socialisation-des-enfants_978926408694413-fr\#.WclzD8jyiM8\#page3

Tenret, Élise (2008). L'école et la Croyance en la Méritocratie. Tesis de doctorado. Dijon: Institut de
Recherche sur l'Éducation. Disponible en: https:// tel.archives-ouvertes.fr/tel-00347360/document Valenzuela, Juan Pablo \& Allende-González, Claudio (2012). Logros en liceos públicos de excelencia en Chile: ¿valor agregado o solo descreme de la élite? Santiago, Chile: Trabajo presentado en el II Congreso Interdisciplinario de Investigación en Educación

Valenzuela, Juan Pablo; Bellei, Cristián \& Los Ríos, Danae de (2010). Segregación escolar en Chile. En Sergio Martinic \& Gregory Elacqua (eds.). ¿Fin de ciclo? Cambios en la gobernanza del sistema educativo, 209-229. Santiago: Oficina Regional de Educación de la UNESCO para América Latina y el Caribe, OREALC-UNESCO, Pontificia Universidad Católica de Chile. Disponible en: http://unesdoc. unesco.org/images/0019/001905/190544S.pdf

Vieira, Maria Manuel (2014). A fabricação da entrada em Medicina. Tensoes, dilemas e suportes. En Leonor Lima Torres \& José Augusto Palhares (eds.). Entre mais e melhor escola em democracia. Inclusão e excelência no sistema educativo português, 49-68. Lisboa: Editora Mundos Sociais.

Villalobos, Cristóbal \& Valenzuela, Juan Pablo (2012). Polarización y cohesión social del sistema escolar chileno. Revista de Análisis Económico, 27 (2), 145-172. Disponible en: http://www.rae-ear.org/ index.php/rae/article/view/366/529

Zanten, Agnès van (2009). Choisir son école. Stratégies familiales et médiations locales. Paris: Presses Universitaires de France, PUF.

Zanten, Agnès van (2015). A Family Affair: Reproducing Elite Positions and Preserving the Ideals of Meritocratic Competition and Youth Autonomy. En Agnès van Zanten, Stephen Ball \& Brigitte Darchy-Koechlin (eds.). Elites, Privilege and Excellence. The National and Global Redefinition of Educational Advantage, 29-42. New York: Routledge. 